\title{
Interdependencies of cellular and humoral immune responses in heterologous and homologous SARS-CoV-2 vaccination
}

\author{
Moritz M. Hollstein ${ }^{1}$, Lennart Münsterkötter ${ }^{1}$, Michael Schön ${ }^{1}$, Armin Bergmann ${ }^{1}$, Thea \\ M. Husar ${ }^{1}$, Anna Abratis ${ }^{1}$, Abass Eidizadeh ${ }^{1}$, Meike Schaffrinski ${ }^{1}$, Karolin Zachmann ${ }^{1}$, \\ Anne Schmitz ${ }^{2}$, Jason S. Holsapple ${ }^{2}$, Hedwig Stanisz-Bogeski ${ }^{1}$, Julie Schanz ${ }^{1}$, Uwe Groß ${ }^{1}$, \\ Andreas Leha ${ }^{1}$, Andreas E. Zautner ${ }^{1}$, Moritz Schnelle ${ }^{1}$, and Luise Erpenbeck ${ }^{1}$ \\ ${ }^{1}$ Universitatsmedizin Gottingen \\ ${ }^{2}$ Universitatsklinikum Munster
}

January 25, 2022

\begin{abstract}
Background: Homologous and heterologous SARS-CoV-2 vaccinations yield different spike protein-directed humoral and cellular immune responses. This study aimed to explore their currently unknown interdependencies. Methods: COV-ADAPT is a prospective, observational cohort study of 417 healthcare workers who received vaccination with homologous ChAdOx1 nCoV-19, homologous BNT162b2 or with heterologous ChAdOx1 nCoV-19/BNT162b2. We assessed humoral (anti-spikeRBD-IgG, neutralizing antibodies, avidity) and cellular (spike-induced $\mathrm{T}$ cell interferon- $\gamma$ release) immune responses in blood samples up to 2 weeks before (T1) and 2 to 12 weeks following secondary immunization (T2). Results: Initial vaccination with ChAdOx1 nCoV-19 resulted in lower anti-spike-RBD-IgG compared to BNT162b2 (70 \pm 114 vs. 226 \pm 279 BAU/ml, p<0.01) at T1. Booster vaccination with BNT162b2 proved superior to ChAdOx1 nCoV-19 at T2 (anti-spike-RBD-IgG: ChAdOx1 nCoV19/BNT162b2 2387 \pm 1627 and homologous BNT162b2 3202 \pm 2184 vs. homologous ChAdOx1 nCoV-19 413 \pm 461 BAU/ml, both $\mathrm{p}<0.001$; spike-induced $\mathrm{T}$ cell interferon- $\gamma$ release: ChAdOx1 nCoV-19/BNT162b2 5069 \pm 6733 and homologous BNT162b2 $4880 \pm 7570$ vs. homologous ChAdOx1 nCoV-19 $1152 \pm 2243 \mathrm{mIU} / \mathrm{ml}$, both $\mathrm{p}<0.001)$. No significant differences were detected between BNT162b2-boostered groups at T2. For ChAdOx1 nCoV-19, no booster effect on $\mathrm{T}$ cell activation could be observed. We found associations between anti-spike-RBD-IgG levels (ChAdOx1 nCoV-19/BNT162b2 and homologous BNT162b2) and T cell responses (homologous ChAdOx1 nCoV-19 and ChAdOx1 nCoV-19/BNT162b2) from T1 to T2. Additionally, anti-spikeRBD-IgG and $\mathrm{T}$ cell response were linked at both time points (all groups combined). All regimes yielded neutralizing antibodies and increased antibody avidity at T2. Conclusions: Interdependencies between humoral and cellular immune responses differ between common SARS-CoV-2 vaccination regimes. T cell activation is unlikely to compensate for poor humoral responses.
\end{abstract}

\section{Hosted file}

Hollstein_et_al_Allergy_Ms.docx available at https://authorea.com/users/452608/articles/ 550624-interdependencies-of-cellular-and-humoral-immune-responses-in-heterologous-andhomologous-sars-cov-2-vaccination 
Figure 1

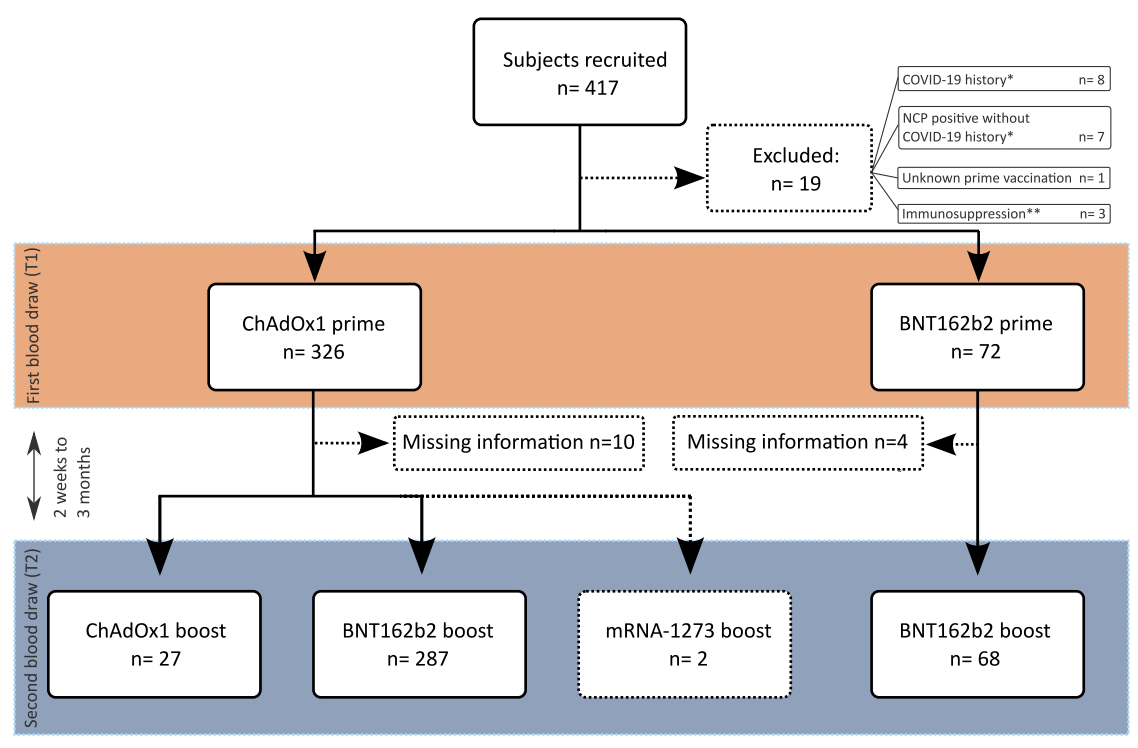

Figure 2
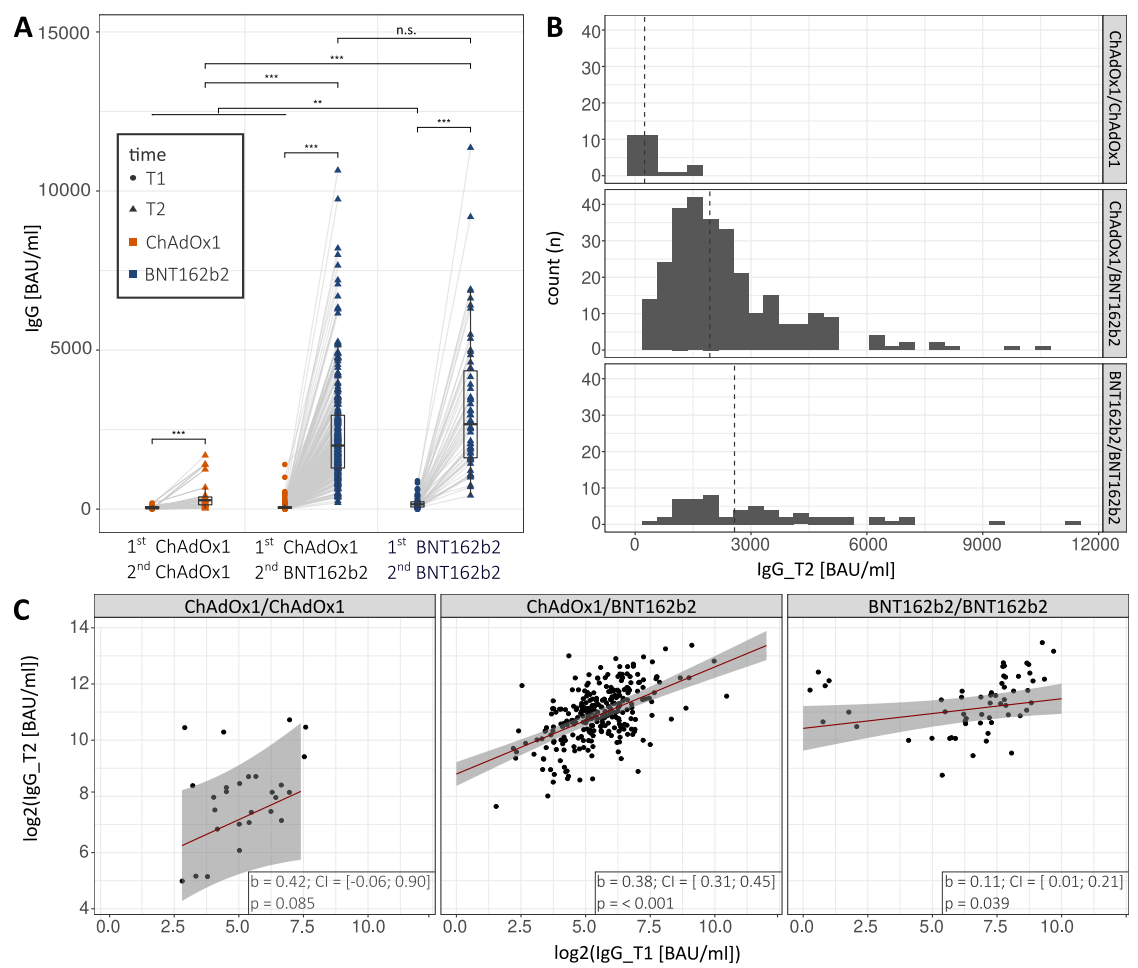
Figure 3
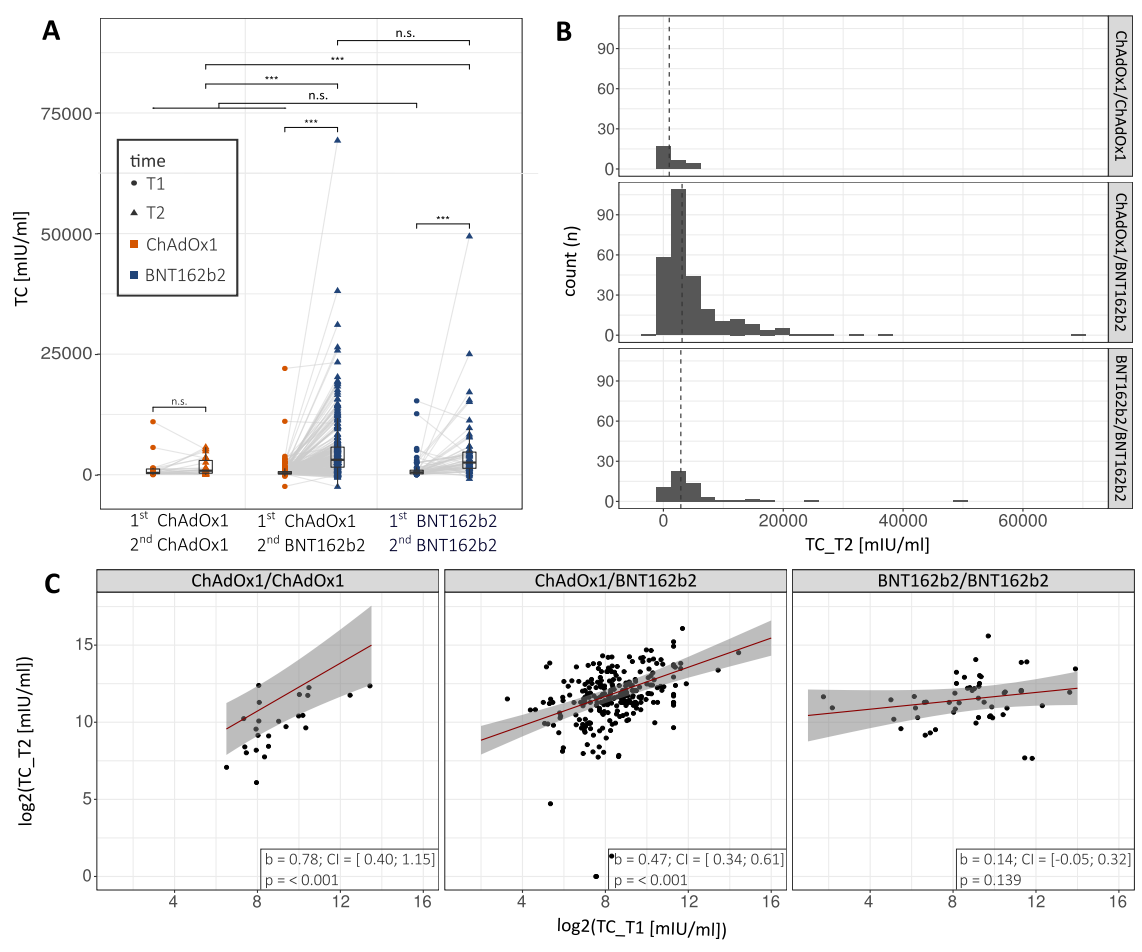

Figure 4
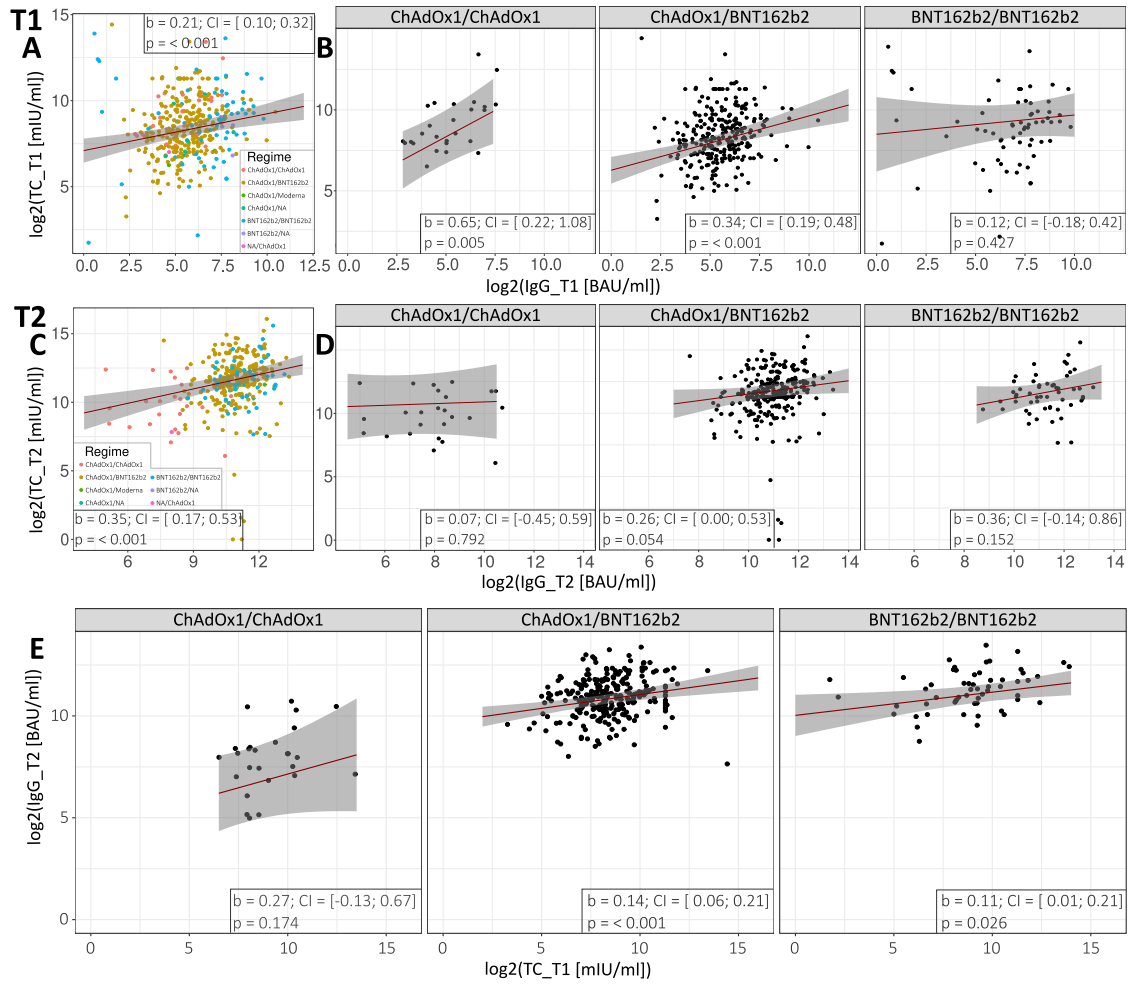
Figure 5

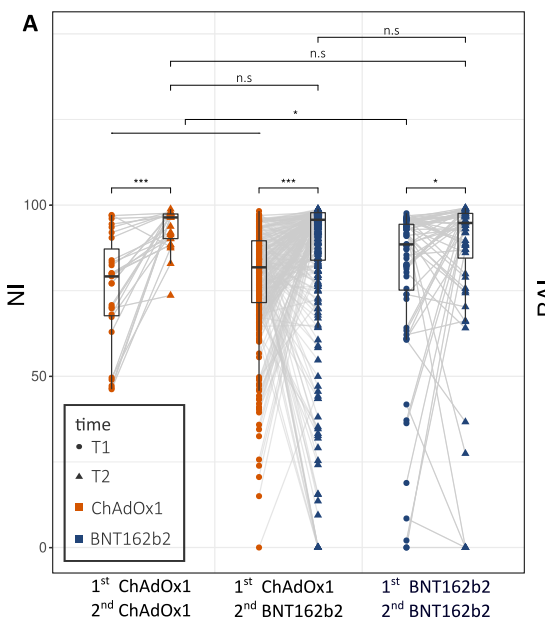

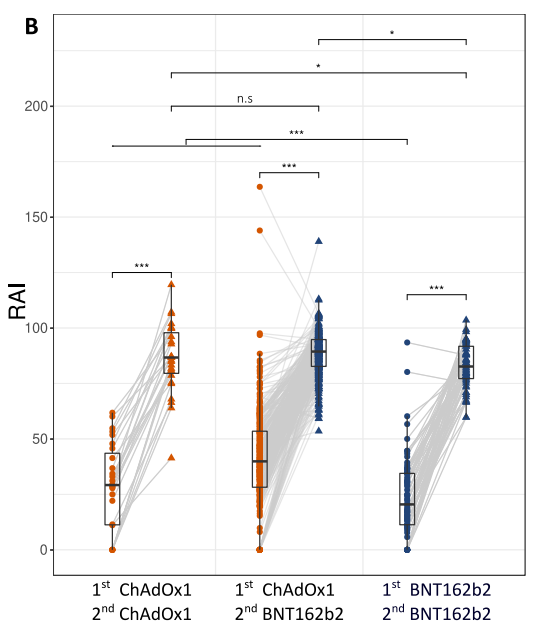

